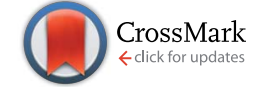

Cite this: RSC Adv., 2017, 7, 1927
Received 5th October 2016

Accepted 8th December 2016

DOI: $10.1039 / c 6 r a 24763 f$

www.rsc.org/advances

\title{
Development and multi-use applications of dengue NS1 monoclonal antibody for early diagnosis
}

\author{
E. Kathiresan, ${ }^{a}$ R. Paramasivan, ${ }^{\text {b V. Thenmozhi, }}$ Aparup Das, ${ }^{b}$ K. J. Dhananjeyan, ${ }^{b}$

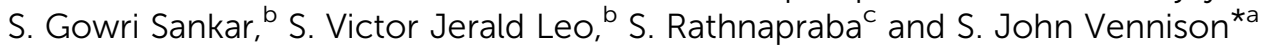

\begin{abstract}
Swift and early diagnosis of dengue is important for case management and epidemiological purpose. We developed a dengue NS1 antigen specific capture enzyme-linked immunosorbent assay (ELISA) for early diagnosis using a panel of monoclonal antibodies (mAbs), which are generated against monomeric nonstructural (NS1) antigen expressed in E. coli. The mAbs were tested for its usefulness in dengue diagnosis during dengue outbreak in Tamil Nadu, India and compared with routinely used methods like RT-PCR, MAC-ELISA and commercially available NS1 antigen detection kit. The results of the comparative analysis suggest that the raised $\mathrm{mAb}$ may be used for the routine screening of suspected sera and also for the detection of dengue virus antigen in field collected vectors (mosquitoes).
\end{abstract}

\section{Introduction}

Dengue is a mosquito-borne viral infection caused by closely related four antigenically distinct virus serotypes (DEN-1, DEN2, DEN-3 and DEN-4) which are classified under the genus Flavivirus, family Flaviviridae. The majority ( $75 \%)$ of infections in humans are asymptomatic or with mild symptoms and may leads to dengue fever (DF) or more severe form known as dengue hemorrhagic fever/dengue shock syndrome (DHF/DSS). Due to non-availability of vaccines or drugs, early diagnosis of dengue infection is important to differentiate between dengue and other diseases with similar clinical symptoms, such as malaria, yellow fever, Japanese encephalitis and chikungunya.,

The dengue virus nonstructural NS1 protein is a $\sim 47 \mathrm{kDa}$ glycoprotein which is produced during viral replication and it has been identified as an important antigen in dengue virus infection. $^{3}$ NS1 protein is produced by all flaviviruses and is secreted from infected mammalian cells during early phases of infection.

NS1 glycoprotein can be detected by an enzyme linked immunosorbent assay (ELISA) using a NS1 glycoprotein specific monoclonal antibody (mAb) in dengue affected human sera. ${ }^{\mathbf{4 - 8}}$ Moreover, this test will provide rapid results, hence it has more advantage on endemic regions. ${ }^{9-13}$ Sensitivity of commercially available kits varies depending on the severity of the cases and the endemic regions, also varies for the four dengue virus serotypes depending on the geographical region. ${ }^{\mathbf{1 4 - 1 6}}$

\footnotetext{
${ }^{a}$ Department of Biotechnology, Anna University, BIT Campus, Tiruchirappalli 620024 , India. E-mail: johnvennison36@gmail.com

${ }^{b}$ Centre for Research in Medical Entomology (CRME), Indian Council of Medical Research, Chinna Chokkikulam, Madurai 625 002, India

${ }^{c}$ Department of Animal Biotechnology, Madras Veterinary College, Tamil Nadu Veterinary and Animal Sciences University, Chennai, 600007, India
}

The virus is transmitted to humans mainly by biting of infected Aedes aegypti and Ae. albopictus. In the absence of vaccine or antiviral treatment epidemiological investigation as well as virus monitoring in mosquitoes is indispensable for implementing effective control of disease spread by reducing mosquito population. Some of the commercially available NS1 antigen based kits, which are intended for human serum analysis, has also been reported to detect dengue antigen in field caught mosquitoes. However, those kits were not evaluated with respect to the sensitivity and stability for the field collected mosquito pools. ${ }^{\mathbf{1} 17}$ NS1 antigen-capture ELISA is more advantages like, sensitive, ease of performing in testing, cost-effective and non-requirement of sophisticated equipment.

In this present study, monoclonal antibodies have been produced against recombinant dengue NS1 antigen. The isotypes of the monoclonal antibodies were determined and specificity and sensitivity of antigen capture ELISA for the detection of dengue infection were determined compared with the commercially available dengue diagnosis kit during on suspected dengue outbreak. The mAbs were utilized in both ELISA and IFA assay to analyse the virus presence in the field collected Ae. aegypti and Ae. albopictus, also in the virus propagated Toxorhynchites splendens larvae for laboratory dengue virus analysis (Toxo-IFA).

\section{Materials and methods}

All experiments were performed in compliance with the relevant laws and institutional guidelines. The study was approved by the ethical committee of the Anna University. Animals were maintained in accordance with the guidelines of the National Institute of Nutrition, Hyderabad, India, and approved by the Institutional Ethical Committee (IEC) of Anna University. 


\section{Anti-dengue NS1 mouse monoclonal hybridomas}

Dengue-2 virus recombinant nonstructural NS1 protein has been expressed in $E$. coli and purified without any in vitro post translational processes. The production and purification of NS1 protein has been described by us already.$^{18}$ Myeloma Sp2/0 cells were obtained from NCCS - Pune, India. Four 6-8 week old female BALB/c mice were immunized 4 times intraperitoneally with $50 \mu \mathrm{g}$ of monomeric NS1 antigen. Complete Freund's adjuvant (Sigma-Aldrich, USA) was used in dose 1 and the incomplete Freund's adjuvant (Sigma-Aldrich, USA) was used in dose 2 and 3. Final dose was immunized with $50 \mu \mathrm{g}$ of NS1 antigen using PBS. The immune response were analyzed using indirect ELISA and serum titers were estimated. ${ }^{19}$ The splenocytes were fused with myeloma $\mathrm{Sp} 2 / 0$ cell line at a ratio of $5: 1$ using $50 \%(\mathrm{w} / \mathrm{v})$ polyethylene glycol (PEG). ${ }^{20}$

\section{Screening and purification of mAbs}

Hybridoma supernatants were screened for the presence of mAbs against dengue NS1 antigen by indirect ELISA. $100 \mu \mathrm{L}$ of purified dengue NS1 protein $\left(10 \mu \mathrm{g} \mathrm{mL}{ }^{-1}\right)$ in PBS was used for coating 96 well plates and incubated overnight at $4{ }^{\circ} \mathrm{C}$. The wells were blocked with $2 \% \mathrm{BSA}$, for $2 \mathrm{~h}$ at $37{ }^{\circ} \mathrm{C}$. After three washes with PBS containing $0.05 \%$ Tween 20 (PBS-T) the wells were incubated with $100 \mu \mathrm{L}$ supernatant from hybridoma clone for $2 \mathrm{~h}$ at $37^{\circ} \mathrm{C}$. The wells were washed three times with PBS and the antibodies were detected using goat anti mouse IgG conjugated with horseradish peroxidase as secondary antibody at a $1: 10000$ dilutions for $1 \mathrm{~h}$ incubation at $37{ }^{\circ} \mathrm{C} .{ }^{21}$ The plate was again washed three times with PBST and $100 \mu \mathrm{L}$ of $3,3^{\prime}, 5,5^{\prime}$ tetramethylbenzidine (TMB) substrate was added to the wells. After $15 \mathrm{~min}$ of color development, optical density was measured at $450 \mathrm{~nm}$. Positive hybridoma cells were cloned by limiting dilution. The isotypes of the mAbs were determined with the mouse monoclonal antibody isotyping kit (Thermo Scientific, USA) according to manufacturer's instructions. The mAbs from hybridoma culture supernatants were purified using Protein-G Sepharose column (Sigma-Aldrich, USA). The affinitypurified mAbs were dialyzed against PBS pH 7.2 for $16 \mathrm{~h}$. Protein estimation was done by Bradford assay. ${ }^{22}$

\section{Toxo-IFA screening}

Dengue serotype 1 (P-23086) virus was obtained from NIV, Pune, India. Tx. splendens larvae were immobilized on ice for few minutes and dengue 1 virus was inoculated intracerebrally with different dilutions. ${ }^{23,24}$ During rearing period, $T x$. splendens larvae were fed with larvae of other species of mosquitoes maintained in the colony and larvae inoculated with sterile BAPS (bovine albumin phosphate buffered saline PH 7.4) were used as controls. Virus inoculated and normal mosquito larvae were grown at $28 \pm 1{ }^{\circ} \mathrm{C}$ and relative humidity of $80 \%$.

After rearing period, head squashes of virus infected and normal larvae were made on the Teflon coated microscopic slides. Slides were air dried and fixed with chilled acetone for 5 minutes. Monoclonal antibody (15 $\mu$ l diluted in PBS $1: 5) /$ cell culture fluid $(20 \mu \mathrm{l})$ was added on the smear and incubated in a humid chamber at $37{ }^{\circ} \mathrm{C}$ for 30 minutes, followed by 4 times wash with $1 \times$ PBS $(20 \mu \mathrm{l}) ; 15 \mu \mathrm{l}$ of $1: 100$ dilution of goat antimouse IgG FITC conjugate (Sigma-Aldrich) was added, incubated for 30 minutes and washed with $1 \times$ PBS for 4 times. The smears were mounted in $10 \%$ glycerol and examined under fluorescent microscope. Commercially available dengue virus specific monoclonal antibodies (Abcam, USA) were used as positive control.

\section{Cross reactivity and specificity of mAbs}

Monoclonal antibodies were coupled with horseradish peroxidase (HRP) according to modified periodate method. ${ }^{25}$ Cross reactivity and specificity of generated mAbs were analyzed by the ELISA and western blot against all four dengue serotypes and also against Japanese encephalitis (JE), West Nile and chikungunya viruses.

\section{NS1 antigen capture ELISA on mosquito pools}

Microtitration plates were coated overnight with $100 \mu \mathrm{l}$ per well of mAb at $4{ }^{\circ} \mathrm{C}$. Wells were washed with PBST buffer (PBS, $0.05 \%$ Tween 20) and $100 \mu \mathrm{l}$ per well of blocking buffer (2\% BSA) was added and incubated at room temperature for $2 \mathrm{~h}$. Mosquito homogenate samples were added to wells (100 $\mu$ l per well) and incubated for $2 \mathrm{~h}$ at room temperature. The wells were washed again and incubated for $2 \mathrm{~h}$ at $37{ }^{\circ} \mathrm{C}$ with $100 \mu \mathrm{l}$ per well of HRP conjugated NS1 mAbs. After three further washes with PBST, $100 \mu \mathrm{L}$ of (TMB) substrate was added to the wells, allowed for color development and optical density was measured at $450 \mathrm{~nm}^{26}$

\section{RNA extraction, RT-PCR and MAC-ELISA}

A total of 30 suspected serum specimens were collected between days 0 and 7 (acute-phase) during on suspected dengue epidemic in Tamil Nadu, India, in 2015. For cross-reactivity analysis, Japanese encephalitis (JE), West Nile virus and chikungunya serum samples (each 5 , total numbers $=15$ ) were analysed. Clinical samples were analyzed using HRP conjugated mAbs and commercially available Panbio Dengue Early ELISA kit (Panbio Diagnostics, Brisbane, Australia) and results were compared. The cut-off value has been calculated using the average absorbance of confirmed negative samples and with their standard deviation. The RNA was extracted from $100 \mu \mathrm{L}$ of serum sample using QIAamp viral RNA mini kit (Qiagen). All the serum samples were subjected to MAC-ELISA and RT-PCR and serotypes were analysed using specific primers. ${ }^{27}$ Selected samples were subjected to real time RT-PCR. ${ }^{28}$

\section{Results}

\section{Production of anti-dengue NS1 mAbs}

Seven positive hybridoma clones were successfully sub-cloned, and three clones (D5, E4 and E7) were selected for further studies based on their reactivity against dengue NS1 protein. All three anti-dengue NS1 mAbs (D5, E4 and E7) had IgG1 type heavy chain and kappa light chain $(\kappa)$. 


\section{Toxo-IFA screening}

In addition to the usual ELISA screening process, we have also screened hybridoma clones using Toxo-IFA system. It is used on the initial screening of hybridoma clones and also on the final confirmation of monoclonal antibody reactivity. This screening method is simple and it has more advantage for screening the reactivity of monoclonal antibodies. The results were also corroborated with the other screening process (Fig. 1A-C). Positivity of virus presence was identified based on the intensity of the fluorescence and no fluorescence were observed on negative control. With the note of this, mAbs D5, E4, and E7 can also be utilized to detect the presence of antigen on IFA assays (unpublished data).

\section{Purification of mAbs}

The purification of mouse mAbs of the IgG1 and the preparation of immune affinity columns were carried out according to the manufacturer's instructions (Sigma-Aldrich, USA). After the purification, antibody concentration for the clones D5, E4 and E7 were $0.598 \mathrm{mg} \mathrm{mL}^{-1}, 0.694 \mathrm{mg} \mathrm{mL}^{-1}$ and $0.612 \mathrm{mg} \mathrm{mL}^{-1}$ respectively.

\section{Specificity of mAbs}

The results showed strong reactivity among all dengue serotypes and no reactivity for JE, West Nile virus and chikungunya. Virus antigen and sera samples were used to analyse the cross reactivity. Specificity of all the three purified mAbs (three clones - D5, E4 and E7) were confirmed.

\section{NS1 antigen capture ELISA}

The NS1 antigen capture ELISA is similar to a standard antigen capture ELISA. The detection limit of antigen concentration was $0.0625 \mu \mathrm{g} \mathrm{mL} \mathrm{m}^{-1}$ and higher sensitivity was observed at $1 \mu \mathrm{g}$ $\mathrm{mL}^{-1}$. The mAbs were also able to identify the NS1 antigen presence on the field collected mosquitoes.

\section{Comparative analysis of different diagnostic methods}

In order to evaluate the efficiency of the mAbs for detection of NS1 antigen in clinical samples, NS1 capturing ELISA has been utilized. The results showed that out of 30 samples, 15 samples were positive on Panbio kit and 19 were positive on mAbs D5, E4 \& E7. However, the samples were further confirmed by RT-PCR and MAC-ELISA and it was found that, out of 30 samples, only 20 samples were positive either on RT-PCR or MAC-ELISA. With reference to the RT-PCR and MAC-ELISA results, out of 20 positive samples, Panbio kit was able to detect 15 samples and mAbs D5, E4 \& E7 were able to detect 19 samples and the specificity was found to be $100 \%$ for both, since it showed no false-positive results (Table 1). The detection sensitivity of D5, E4 \& E7 was consistent from the samples collected from the onset of illness (0-7 days) (Fig. 2-results shown here for the clone D5 only) and no cross-reactivity was observed with JE, WNV and CHIKV samples. Serotypes were determined for all the positive samples using RT-PCR and for RT-PCR negative
1.A. Positive control - Commercial mAb(Abcam, USA)

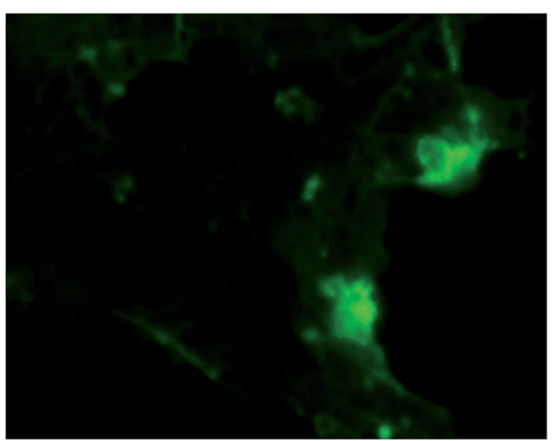

1.B. mAb (D5)

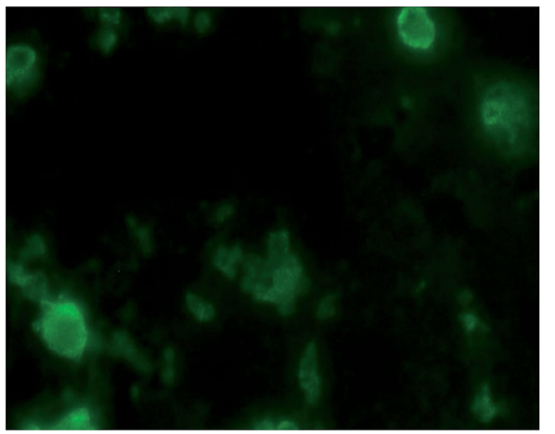

\section{C. Negative Control - Normal Toxo larvae}

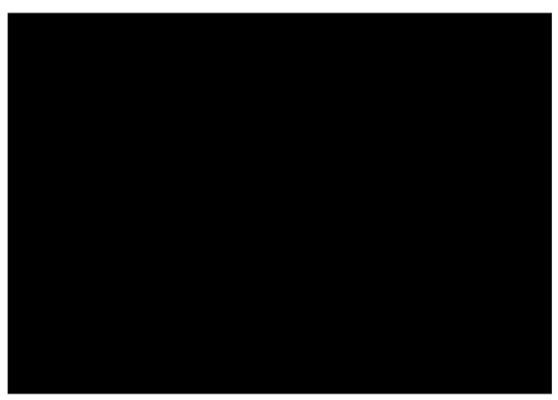

Fig. 1 Toxo-IFA screening. (A \& B) Fluorescence indicates the presence of dengue virus. (C) No fluorescence were observed on negative control.

samples (sample ID: NG248, NG246, NG256 \& NG268) real time RT-PCR was used to determine the serotypes. ${ }^{27,28}$

\section{Discussion}

Monoclonal antibodies have become inevitable components in diagnostic field. It is promising on disease diagnostic field. ${ }^{29-33}$ The present work focuses on the determination of possible use of developing an antigen capture assay for early diagnosis of acute dengue virus infections. Three monoclonal antibodies producing clones (D5, E4 \& E7) have been raised against the dengue virus recombinant NS1 antigen.

NS1 antigen capture ELISA has distinct diagnosing potentials than any other diagnosis process. Several studies have been reported using the NS1 antigen detection method for the early 
Table 1 Suspected serum samples collected from the patients with clinical dengue symptoms on different diagnostic methods (positive samples on either RT-PCR or MAC-ELISA $=20)(n=30)$

\begin{tabular}{|c|c|c|c|c|c|c|c|}
\hline Sl no & Sample ID & $\begin{array}{l}\text { Days from the onset } \\
\text { of illness }\end{array}$ & RT-PCR & MAC-ELISA & Panbio & D5 mAb & Serotype \\
\hline 1 & NG209 & 0 & + & - & + & + & DEN1 \\
\hline 2 & NG217 & 0 & + & - & + & + & DEN4 \\
\hline 3 & NG251 & 0 & + & - & + & + & DEN2 \\
\hline 4 & NG215 & 1 & + & - & + & + & DEN3 \\
\hline 5 & NG261 & 1 & + & - & - & + & DEN1 \\
\hline 6 & NG227 & 2 & + & + & + & + & DEN1 \\
\hline 7 & $\mathrm{NG} 248^{a}$ & 2 & - & + & + & + & DEN3 \\
\hline 8 & NG265 & 2 & + & + & - & + & DEN3 \\
\hline 9 & NG206 & 3 & + & + & + & + & DEN2 \\
\hline 10 & NG243 & 3 & + & + & + & + & DEN3 \\
\hline 11 & $\mathrm{NG}_{246^{a}}$ & 3 & - & + & - & + & DEN1 \\
\hline 12 & NG262 & 3 & + & + & + & + & DEN1 \\
\hline 13 & NG200 & 4 & + & + & + & + & DEN2 \\
\hline 14 & NG222 & 5 & + & + & + & + & DEN2 \\
\hline 15 & NG240 & 5 & + & + & + & + & DEN1 \\
\hline 16 & NG242 & 6 & + & + & + & + & DEN1 \\
\hline 17 & NG210 & 7 & + & + & - & + & DEN3 \\
\hline 18 & $\mathrm{NG} 256^{a}$ & 7 & - & + & + & + & DEN2 \\
\hline 19 & NG268 ${ }^{a}$ & 7 & - & + & - & - & DEN4 \\
\hline 20 & NG275 & 7 & + & + & + & + & DEN2 \\
\hline Total & & & 16 & 15 & 15 & 19 & \\
\hline
\end{tabular}

diagnosis of dengue virus infection, however still the sensitivity and competency of NS1 antigen based diagnosis need more evaluation. ${ }^{34,35}$ The sensitivity of our mAbs (D5, E4 \& E7) were up to $0.0625 \mu \mathrm{g} \mathrm{mL}{ }^{-1}$ on NS1 antigen detection.

The mAbs showed no cross reactivity with other closely related members of the genus Flavivirus (Japanese encephalitis (JE), West Nile virus) and chikungunya virus. In addition, the

\section{Sensitivity of RT-PCR, MAC-ELISA, Panbio and mAb D5}

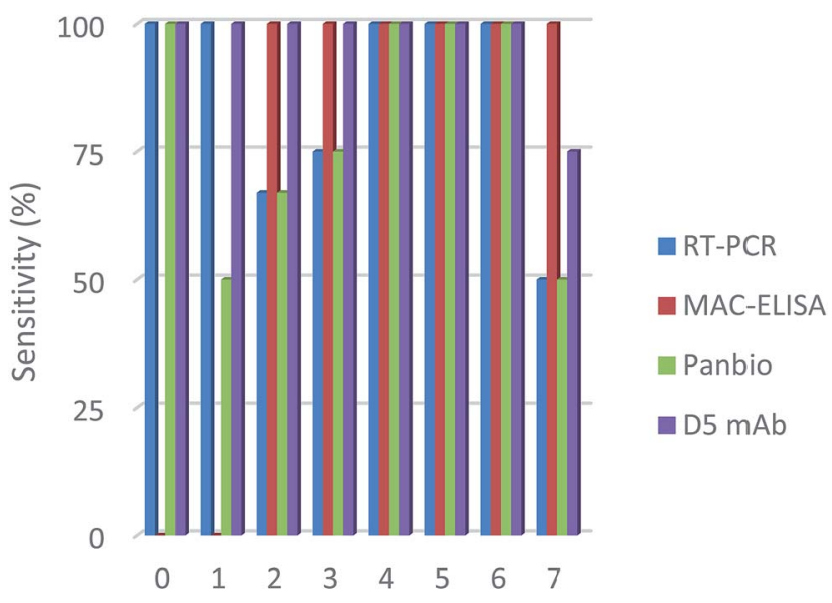

Number of days after onset of illness

Fig. 2 NS1 antigen detection sensitivity percentage using different diagnosis methods on number of days after onset of illness. detecting sensitivity of dengue serotypes is almost similar. While comparing with Panbio kit, the results were better (Panbio $75 \%$ and mAbs D5, E4 \& E7 95\%). It is found that results varied with Panbio and the mAbs (D5, E4 \& E7) for the clinical samples NG210, NG265. To analyze the possible reason behind this, we serotyped all the samples and found that both NG210 and NG265 were serotype 3. This is consistent with earlier observations reporting Panbio kit has low sensitivity on detecting dengue 3 serotype. ${ }^{36-38}$

Till date, diagnostic kits intended for the detection of WNV and Saint Louis encephalitis virus (SLEV) are available commercially. No such kits are available for DENV detection in mosquitoes. A handful of researchers have shown that commercially available dengue NS1 antigen detection kit intended for human serum can also be useful for antigen detection in mosquitoes (Dengue NS1 Ag Strip, Bio-Rad France, Panbio Dengue Early ELISA; Panbio, Australia). ${ }^{39}$ Though the Platelia Dengue NS1 Ag kit has reported 98\% sensitivity for detecting DENV in mosquito pools, the application of the test in field conditions still in doubt since the sensitivity was assessed in experimentally infected mosquitoes in lab. ${ }^{40}$ Panbio kit also reported with better detection sensitivity on infected mosquitoes. ${ }^{41}$ Our mAbs showed $100 \%$ sensitivity when analyzed with field collected mosquitoes (data not shown). The ability of mAbs were also checked in detecting NS1 antigen in dengue virus infected Tx. splendens larvae using Toxo-IFA system.

\section{Conclusions}

In this study, we have developed a panel of anti-NS1 mAb secreting hybridoma clones and validated the use of mAbs (D5, 
E4 \& E7) as a diagnostic tool for dengue diagnosis during outbreak investigation. The mAbs were successfully utilized in ELISA and immunofluorescence assay to analyse the virus presence in field caught Ae. aegypti, Ae. albopictus and in virus propagated Tx. splendens larvae (Toxo-IFA).

\section{Acknowledgements}

This work was supported by the Department of Science Technology (SERB) from the Government of India, Grant No. SR/SO/ HS-0188/2012. The authors would like to thank Mr R. Sathish Babu, Mr T. Balaji (Centre for Research in Medical Entomology (CRME), Indian Council of Medical Research) and Ms. S. Saranya (Madras Veterinary College, Tamil Nadu Veterinary and Animal Sciences University) for their technical support.

\section{References}

1 H. Palanivel, S. Nair, A. Subramaniyan, P. V. J. Ratnam and R. Kanungo, Indian J. Pathol. Bacteriol., 2015, 58(3), 328-331.

2 S. Gowri Sankar, K. J. Dhananjeyan, R. Paramasivan, V. Thenmozhi and B. K. Tyagi, Clin. Microbiol. Infect., 2012, 18(1), E8-E10.

3 P. R. Young, P. A. Hilditch, C. Bletchly and W. Halloran, J. Clin. Microbiol., 2000, 38, 1053-1057.

4 S. Alcon, A. Talarmin, M. Debruyne, A. Falconar, V. Deubel and M. Flamand, J. Clin. Microbiol., 2002, 40, 376-381.

5 C. H. Huang, L. L. Kuo, K. D. Yang, P. S. Lin, P. L. Lu, C. C. Lin, K. Chang, T. C. Chen, W. R. Lin, C. Y. Lin, Y. H. Chen and H. S. Wu, J. Microbiol., Immunol. Infect., 2013, 4, 358-365.

6 F. M. Kassim, M. N. Izati, T. A. TgRogayah, Y. M. Apandi and Z. Saat, Southeast Asian J. Trop. Med. Public Health, 2011, 42(3), 562-569.

7 C. Puttikhunt, T. Prommool, N. U-thainual, P. Ongajchaowlerd, K. Yoosook, C. Tawilert, T. Duangchinda, A. Jairangsri, N. Tangthawornchaikul, P. Malasit and w. Kasinrerk, J. Clin. Virol., 2011, 50, 314-319.

8 S. Datta and C. Wattal, Indian J. Med. Microbiol., 2010, 28, 107-110.

9 A. K. Falconar, Clin. Vaccine Immunol., 2008, 15, 549-561.

10 A. K. Falconar, Virol. J., 2013, 10, 126.

11 P. Koraka, C. P. Burghoorn-Maas, A. Falconar, T. E. Setiati, K. Djamiatun, J. Groen and A. D. J. Osterhaus, J. Clin. Microbiol., 2003, 41, 4154-4159.

12 R. Lima Mda, R. M. Nogueira, A. M. Filippis, P. C. Nunes, C. C. Sousa, M. H. Silva and F. B. Santos, J. Virol. Methods., 2014, 204, 105-108.

13 M. L. Moi, T. Omatsu, S. Tajima, C. K. Lim, A. Kotaki, M. Ikeda, F. Harada, M. Ito, M. Saijo, I. Kurane and T. Takasaki, J. Trav. Med., 2013, 20(3), 185-193.

14 J. Barniol, R. Gaczkowsk, E. V. Barbato, R. V. da Cunha, D. Salgado, E. Martinez, C. S. Segarra, E. B. Pleites Sandoval, A. Mishra, I. S. Laksono, C. S. Lum, J. G. Martínez, A. Núnez, A. Balsameda, I. Allende, G. Ramírez, E. Dimaano, K. Thomacheck, N. A. Akbar, E. E. Ooi, E. Villegas, T. T. Hien, J. Farrar, O. Horstick, A. Kroeger and T. Jaenisch, BMC Infect. Dis., 2011, 11, 106.
15 J. S. Castleberry and C. R. Mahon, Clin. Lab. Sci., 2003, 16, 34-38.

16 S. Chaterji, J. C. Allen Jr, A. Chow, Y. Leo and E. Ooi, Am. J. Trop. Med. Hyg., 2011, 84(2), 224-228.

17 D. Y. Chao, Y. J. Liu, W. F. Shen, W. C. Tu, J. U. Galula and H. C. Wu, Vector Borne and Zoonotic Diseases, 2015, 52, 134-141.

18 S. Gowri Sankar, K. J. Dhanajeyan, R. Paramasivan, V. Thenmozhi, B. K. Tyagi and S. John Vennison, BioMed Res. Int., 2013, 8, 343195.

19 X. X. Ding, B. DI and X. Y. Che, Xibao Yu Fenzi Mianyixue Zazhi, 2011, 27(7), 777-779.

20 G. Kohler and C. Milstein, Nature, 1975, 256, 495-497.

21 P. Perrin, Laboratory techniques in rabies, 4th edn, World

Health Organization, Geneva, 1996, pp. 433-444.

22 M. M. Bradford, Anal. Biochem., 1976, 72, 248-254.

23 D. T. Mourya, Trans. R. Soc. Trop. Med. Hyg., 1990, 84, 580. 24 S. Norazizah and A. Sazaly, J. Entomol., 2006, 3(1), 89-94.

25 G. B. Wisdom, Methods in molecular biology immunochemical protocols, 3 edn, Humana Press Inc., 2005, pp. 127-130.

26 R. Rajendran, A. Gajanaana and P. Samuel, Annual Report, 1992-93, Centre for Research in Medical Entomology, Madurai, Tamil nadu, 1992, pp. 4-11.

27 R. S. Lanciotti, C. H. Calisher, D. J. Gubler, G. J. Chang and A. V. Vorndam, J. Clin. Microbiol., 1992, 30, 545-551.

28 B. W. Johnson, B. J. Russell and R. S. Lanciotti, J. Clin. Microbiol., 2005, 43(10), 4977-4983.

29 C. Zanluca, G. Mazzarotto, J. Bordignon and C. Santos, PLoS One, 2014, 9(11), e110620.

30 Y. Tang, C. Chiu, C. Lin, C. Huang, Y. Chen, R. Destura, D. Chao and H. Wu, Int. J. Mol. Sci., 2015, 16(11), 27850-27864.

31 A. Ganguly, R. B. Malabadi, P. K. Bhatnagar, X. Tang, D. Das, R. Loebenberg, M. R. Suresh and H. H. Sunwoo, J. Viro. Methods., 2015, 220, 5-12.

32 S. H. Kim, Y. N. Kim, T. T. Truong, N. T. Thu Thuy, Q. Mai le and Y. S. Jang, Biochem. Biophys. Res. Commun., 2016, 473, 894-898.

33 L. Jihoo, K. Hak-Yong, C. Chom-Kyu and S. Hyun-Ok, Diagn. Microbiol. Infect. Dis., 2015, 82, 128-134.

34 T. Gelanew, B. K. Poole-Smith and E. Hunsperger, J. Virol. Methods., 2015, 222, 214-223.

35 A. Fatima, H. Wang, K. Kang, L. Xia, Y. Wang, W. Ye, J. Wang and X. Wang, PLoS One, 2014, 9(4), e95263.

36 L. L. Hermann, B. Thaisomboonsuk, Y. Poolpanichupatam, R. G. Jarman, S. Kalayanarooj, A. Nisalak, I. K. Yoon and S. Fernandez, PLoS Neglected Trop. Dis., 2014, 8(10), e3193.

37 M. Lima, R. Nogueira, H. Schatzmayr and F. Santos, PLoS Neglected Trop. Dis., 2010, 4(7), e738.

38 M. Lima, R. Nogueira, H. Schatzmayr, A. M. Filippis and F. Santos, Clin. Vaccine Immunol., 2011, 18(6), 1031-1033.

39 N. Voge, I. Sánchez-Vargas, C. Blair, L. Eisen and B. Beaty, Am. J. Trop. Med. Hyg., 2013, 88(2), 260-266.

40 T. Cheong-Huat, W. Pei-Sze Jeslyn, L. Mei-Zhi, V. Indra and N. Lee-Ching, Vector-Borne and Zoonotic Diseases, 2011, 11(6), 789-792.

41 D. A. Muller, F. D. Frentiu, A. Rojas, L. A. Moreira, S. L. O'Neill and P. R. Young, J. Virol. Methods, 2012, 183, 90-93. 